\title{
Effect of Erythropoietin Therapy on the Lipid Profile in Patients with Chronic Kidney Disease - A Single Center Study
}

\author{
Ali J Hashim Al-Saedi ${ }^{1 *}$, Sabah N Alwachi ${ }^{2}$ and Abdul Hakeem A Karim ${ }^{3}$ \\ ${ }^{1}$ Nephrologist-Al kindy College of Medicine, University of Baghdad, Iraq \\ ${ }^{2}$ Professor of Physiology, Department of Biology, College of Science, University of Baghdad, Iraq
}

${ }^{3}$ Biologist, College of Basic Education, Al Mustansiriya University, Iraq

\begin{abstract}
Dyslipidemia is common in chronic kidney disease patients and alterations in serum lipid profile vary widely depending on the level of kidney function. We studied two hundred Iraqi patients who were diagnosed as having chronic kidney disease from June 2011 to January 2013. They were given erythropoietin for seven months. One hundred sixty patients $(80 \%)$ showed response to treatment with an improvement in lipid profile, while the other 40 patients $(20 \%)$ showed no significant response to treatment. In conclusion, treatment with erythropoietin seems to positively influence the lipid profile in patients with chronic kidney disease.
\end{abstract}

Keywords: Chronic kidney disease; Epoetin; Lipids

\section{Introduction}

Patients with chronic kidney disease (CKD) are at higher risk of cardiovascular disease $(\mathrm{CAD})$ than subjects in the general population [1-3]. Dyslipidemia is common in CKD patients and alterations in serum lipid profile vary widely depending on the level of kidney function and the degree of proteinuria [4-6]. Patients frequently have elevated serum total cholesterol (TC) and low-density lipoprotein cholesterol (LDL-C) levels. The classic lipid profile of late-stage CKD includes increased triglyceride (TG) levels, moderately elevated or normal LDL cholesterol levels and low high density lipoprotein cholesterol (HDL-C) levels $[4,5,7]$.

The reduction in serum levels of HDL-C represents an important lipoprotein abnormality in patients with decreased renal function [59]. Low levels of HDL-C have also been shown to be an independent risk factor for $\mathrm{CAD}$ and premature atherosclerosis regardless of serum LDL-C and TG levels $[10,11]$. HDL-C is a potent anti atherogenic lipoprotein and recent observational, biological and clinical evidence strongly suggests that HDL-C is a promising target of therapeutic intervention $[12,13]$.

\section{Material and Methods}

From June 2011 to January 2013, a total of 200 patients (100 males $\& 100$ females) were studied. Those patients were seen in the department of nephrology in Al-Kindy Teaching Hospital, Baghdad / Iraq. The age

\begin{tabular}{|c|c|c|c|}
\hline $\begin{array}{l}\text { Groups } \\
\text { Months }\end{array}$ & $\begin{array}{c}\text { Mild } \\
\text { (NO:36) } \\
\text { Mean } \pm \text { S.D. }\end{array}$ & $\begin{array}{c}\text { Moderate } \\
\text { (NO:62) } \\
\text { Mean } \pm \text { S.D. }\end{array}$ & $\begin{array}{c}\text { Severe } \\
\text { (NO:42) } \\
\text { Mean } \pm \text { S.D. }\end{array}$ \\
\hline M1 & $\begin{array}{c}10.18 \pm 0.26 \\
B C\end{array}$ & $\begin{array}{c}8.59 \pm 0.45 \\
A C\end{array}$ & $\begin{array}{c}6.54 \pm 0.39 \\
A B\end{array}$ \\
\hline M2 & $\begin{array}{c}10.57 \pm 0.59 \text { ** } \\
B C\end{array}$ & $\begin{array}{c}9.35 \pm 0.44^{* *} \\
\mathrm{AC}\end{array}$ & $\begin{array}{c}8.14 \pm 0.91 \text { ** } \\
A B\end{array}$ \\
\hline M3 & $\begin{array}{c}10.91 \pm 0.48^{* *} \\
B C\end{array}$ & $\begin{array}{c}9.91 \pm 0.57^{* *} \\
\text { AC }\end{array}$ & $\begin{array}{c}8.84 \pm 1.03 \text { ** } \\
A B\end{array}$ \\
\hline M4 & $\begin{array}{c}11.45 \pm 0.53^{* *} \\
B C\end{array}$ & $\begin{array}{c}10.56 \pm 0.63 \text { ** } \\
\text { AC }\end{array}$ & $\begin{array}{c}9.48 \pm 0.98 \text { ** } \\
A B\end{array}$ \\
\hline M5 & $\begin{array}{c}12.00 \pm 0.53 \text { ** } \\
B C\end{array}$ & $\begin{array}{c}11.09 \pm 0.74 \text { ** } \\
\text { AC }\end{array}$ & $\begin{array}{c}10.19 \pm 0.88 \text { ** } \\
A B\end{array}$ \\
\hline M6 & $\begin{array}{c}12.56 \pm 0.48 \text { ** } \\
B C\end{array}$ & $\begin{array}{c}11.67 \pm 0.78 \text { ** } \\
\text { AC }\end{array}$ & $\begin{array}{c}10.89 \pm 0.88 \text { ** } \\
A B\end{array}$ \\
\hline M7 & $\begin{array}{c}13.29 \pm 0.65^{* *} \\
B C\end{array}$ & $\begin{array}{c}12.23 \pm 0.77^{* *} \\
A C\end{array}$ & $\begin{array}{c}11.58 \pm 0.93^{* *} \\
A B\end{array}$ \\
\hline
\end{tabular}

Table 1: Comparison of $\mathrm{Hb}$ between rhEPO responders patients. ranged from 45-75 years, all patients were diagnosed as having renal anemia from history, clinical examination and laboratory investigations including lipid profile TC, TG and HDL-C. Patients were given epoetin alfa (4000 IU), subcutaneously (SC) once, twice or three times a week, depending on the degree of anemia. All patients received atorvastatin $10 \mathrm{mg}$ once daily along with restricted protein diet. All patients were not on dialysis.

\section{Statistical Analysis}

The Statistical Analysis System-SAS was used to detect the difference factors in study parameters (applied of completely randomized designCRD). Duncan's test was used to find the difference factors in study parameters. ${ }^{*}$ : Significant at level $\mathrm{P}<0.05 /{ }^{* *}$ : Highly Significant at level $\mathrm{P}<0.01 /$
A: Significant mild group with other groups at level $\mathrm{P}<0.05 /$
B: Significant moderate group with other groups at level $\mathrm{P}<0.05 /$
C: Significant severe group with other groups at level $\mathrm{P}<0.05$.

\section{Results}

In this study200 CKD patients with renal anemia were given EPO treatment (4000 IU) SC. Patients depending on the response to EPO therapy were classified into two groups, rhEPO responders 160 patients (80\%) and rhEPO non-responders 40 patients (20\%), the two groups were divided into three sub-groups depending on degree of anemia according to the value of hemoglobin $(\mathrm{Hb})$, was as follow: rhEPO responders mild anemia $(\mathrm{Hb} \geq 10 \mathrm{~g} / \mathrm{dl})$ : four times (Month (M)1-M5) (Table 1 and 2) and two times (M6-M7) per month, moderate anemia $(8 \mathrm{~g} / \mathrm{dL} \leq \mathrm{Hb}<10 \mathrm{~g} / \mathrm{dL})$ : four times (M1-M2), eight times (M3-M6) and four times (M7) per month and for those with severe anemia $(\mathrm{Hb}<8$ $\mathrm{g} / \mathrm{dL}$ ): eight times (M2-M6) and four times (M7) per month. For those

*Corresponding author: Ali J Hashim Al-Saedi, Faculty of Medicine-Al Kindy College of Medicine, University of Baghdad, Iraq, E-mail: dralijasm@yahoo.com

Received August 16, 2013; Accepted September 17, 2013; Published September 21,2013

Citation: Al-Saedi AJH, Alwachi SN, Karim AHA(2013) Effect of Erythropoietin Therapy on the Lipid Profile in Patients with Chronic Kidney Disease - A Single Center Study. J Nephrol Ther 3: 140. doi:10.4172/2161-0959.1000140

Copyright: (c) $2013 \mathrm{Al}$-Saedi AJH, et al. This is an open-access article distributed under the terms of the Creative Commons Attribution License, which permits unrestricted use, distribution, and reproduction in any medium, provided the original author and source are credited. 


\begin{tabular}{|c|c|c|c|}
\hline $\begin{array}{l}\text { Groups } \\
\text { Months }\end{array}$ & $\begin{array}{c}\text { Mild } \\
\text { (NO:36) } \\
\text { Mean } \pm \text { S.D. }\end{array}$ & $\begin{array}{c}\text { Moderate } \\
\text { (NO:62) } \\
\text { Mean } \pm \text { S.D. }\end{array}$ & $\begin{array}{c}\text { Severe } \\
\text { (NO:42) } \\
\text { Mean } \pm \text { S.D. }\end{array}$ \\
\hline M1 & $\begin{array}{c}32.56 \pm 0.84 \\
B C\end{array}$ & $\begin{array}{c}27.84 \pm 1.36 \\
B C\end{array}$ & $\begin{array}{c}21.57 \pm 1.23 \\
A B\end{array}$ \\
\hline M2 & $\begin{array}{c}33.78 \pm 1.89 \text { ** } \\
\text { BC }\end{array}$ & $\begin{array}{c}30.11 \pm 1.34 \text { ** } \\
\text { BC }\end{array}$ & $\begin{array}{c}26.47 \pm 2.69 \text { ** } \\
A B\end{array}$ \\
\hline M3 & $\begin{array}{c}34.78 \pm 1.42 \text { ** } \\
\text { BC }\end{array}$ & $\begin{array}{c}31.81 \pm 1.74 \text { BC } \\
\text { ** }\end{array}$ & $\begin{array}{c}28.62 \pm 3.09 \text { ** } \\
A B\end{array}$ \\
\hline M4 & $\begin{array}{c}36.44 \pm 1.59 \text { ** } \\
\text { BC }\end{array}$ & $\begin{array}{c}33.74 \pm 1.90 \text { ** } \\
\text { BC }\end{array}$ & $\begin{array}{c}30.48 \pm 2.91 \text { ** } \\
A B\end{array}$ \\
\hline M5 & $\begin{array}{c}38.00 \pm 1.51 \text { ** } \\
B C\end{array}$ & $\begin{array}{c}35.32 \pm 2.23 \text { ** } \\
\mathrm{BC}\end{array}$ & $\begin{array}{c}32.67 \pm 2.66 \text { ** } \\
A B\end{array}$ \\
\hline M6 & $\begin{array}{c}39.78 \pm 1.46 \text { ** } \\
\text { BC }\end{array}$ & $\begin{array}{c}37.09 \pm 2.32 \text { ** } \\
\text { BC }\end{array}$ & $\begin{array}{c}34.71 \pm 2.69 \text { ** } \\
A B\end{array}$ \\
\hline M7 & $\begin{array}{c}41.83 \pm 1.92 \text { ** } \\
B C\end{array}$ & $\begin{array}{c}38.74 \pm 2.35 \text { ** } \\
\mathrm{BC}\end{array}$ & $\begin{array}{c}36.76 \pm 2.88 \text { ** } \\
A B\end{array}$ \\
\hline
\end{tabular}

Table 2: Comparison of Hct between rhEPO responder patients.

\begin{tabular}{|c|c|c|c|}
\hline $\begin{array}{l}\text { Groups } \\
\text { Months }\end{array}$ & $\begin{array}{c}\text { Mild } \\
\text { (NO:36) } \\
\text { Mean } \pm \text { S.D. }\end{array}$ & $\begin{array}{c}\text { Moderate } \\
\text { (NO:62) } \\
\text { Mean } \pm \text { S.D. }\end{array}$ & $\begin{array}{c}\text { Severe } \\
\text { (NO:42) } \\
\text { Mean } \pm \text { S.D. }\end{array}$ \\
\hline M1 & $\begin{array}{c}240.53 \pm 18.39 \\
B C\end{array}$ & $\begin{array}{c}225.32 \pm 20.54 \\
\text { AC }\end{array}$ & $\begin{array}{c}168.10 \pm 14.24 \\
A B\end{array}$ \\
\hline M2 & $\begin{array}{c}221.67 \pm 20.94 * * \\
C\end{array}$ & $\begin{array}{c}213.52 \pm 24.04 \text { * } \\
\text { ** }\end{array}$ & $\begin{array}{c}162.26 \pm 14.24 \\
A B\end{array}$ \\
\hline M3 & $\begin{array}{c}209.83 \pm 25.23 \text { ** } \\
C\end{array}$ & $\begin{array}{c}209.05 \pm 23.46 \text { ** } \\
C\end{array}$ & $\begin{array}{c}159.81 \pm 13.71 \text { ** } \\
A B\end{array}$ \\
\hline M4 & $\begin{array}{c}199.56 \pm 27.07 \text { * } \\
\text { ** }\end{array}$ & $\begin{array}{c}205.29 \pm 23.22 \text { ** } \\
C\end{array}$ & $\begin{array}{c}158.67 \pm 12.85 \text { ** } \\
A B\end{array}$ \\
\hline M5 & $\begin{array}{c}194.78 \pm 26.91 \text { ** } \\
C\end{array}$ & $\begin{array}{c}199.23 \pm 21.55^{* *} \\
C\end{array}$ & $\begin{array}{c}157.02 \pm 11.77^{* *} \\
A B\end{array}$ \\
\hline M6 & $\begin{array}{c}186.39 \pm 24.43^{* *} \\
C\end{array}$ & $\begin{array}{c}194.56 \pm 21.26 \text { ** } \\
C\end{array}$ & $\begin{array}{c}154.93 \pm 11.90 \text { ** } \\
A B\end{array}$ \\
\hline M7 & $\begin{array}{c}180.17 \pm 20.80 \text { ** } \\
B C\end{array}$ & $\begin{array}{c}190.18 \pm 21.77^{* *} \\
\text { AC }\end{array}$ & $\begin{array}{c}154.12 \pm 10.83 \text { ** } \\
A B\end{array}$ \\
\hline
\end{tabular}

Table 3: Comparison of cholesterol between rhEPO responders patients.

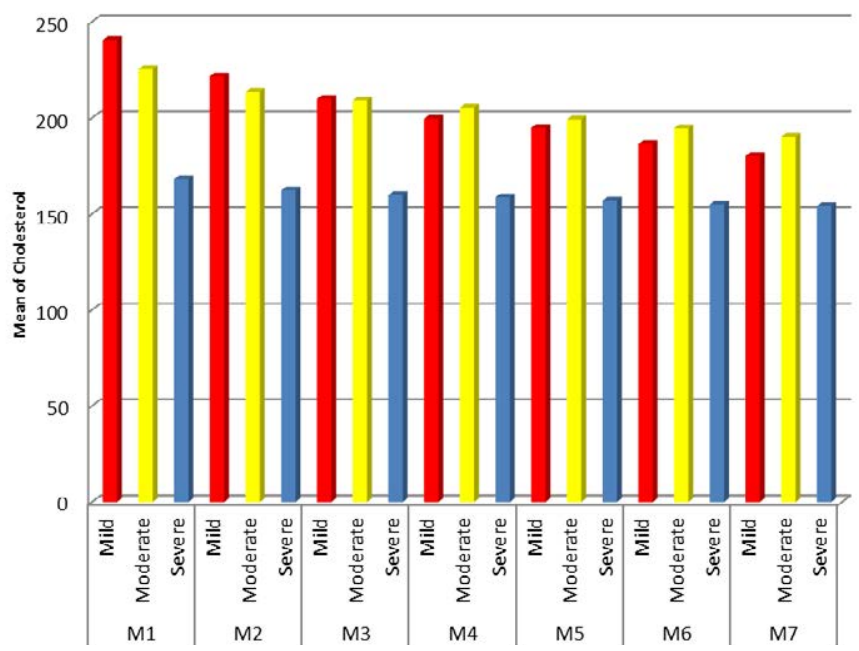

Figure 1: Comparison of cholesterol between rhEPO responders patients.

who were rhEPO non- responders mild anemia: four times (M1-M4), eight times (M5-M6) and twelve times (M7) per month, moderate anemia: four times (M1-M2), eight times (M3-M5) and twelve times (M6-M7) per month and those with severe anemia: eight times (M1M4) and twelve times (M5-M7) per month. EPO dose (4000 IU syringe
$\mathrm{SC})$. In the rhEPO responders results showed increase in $\mathrm{Hb}(\mathrm{g} / \mathrm{dl})$ haematocrit $(\mathrm{Hct})(\%)$ and HDL-C $(\mathrm{mg} / \mathrm{dl})$ with decrease in TC $(\mathrm{mg} /$ $\mathrm{dl})$, TG (mg/dl) levels during the period of EPO therapy (Table 3 and Figure 1) showed significant difference $(\mathrm{P}<0.05)$ in the mean TC $(\mathrm{mg} /$ dl) of mild (M1\& M7), moderate (M1\&M7) and severe (M1\&M7) anaemia groups were $(240.53 \pm 18.39 \& 180.17 \pm 20.80),(225.32 \pm 20.54$ $\& 190.18 \pm 21.77)$ and $(168.10 \pm 14.24 \& 154.12 \pm 10.83)$ respectively. While contrast significant differences were observed for the other month among all anaemia groups. The levels of TC $(\mathrm{mg} / \mathrm{dl})$ before therapy (M1) and after therapy (M2-M7) showed significant difference $(\mathrm{P}<0.01)$ in mild anaemia $(240.53 \pm 18.39)$ and $(221.67 \pm 20.94 \mathrm{M} 2-$ $180.17 \pm 20.80 \mathrm{M} 7)$, moderate anaemia $(225.32 \pm 20.54)$ and $(213.52$ $\pm 24.04 \mathrm{M} 2-190.18 \pm 21.77 \mathrm{M} 7)$ and severe anaemia groups $(168.10 \pm$ $14.24)$ and $(162.26 \pm 14.24 \mathrm{M} 2-154.12 \pm 10.38 \mathrm{M} 7)$ respectively.

(Table 4 and Figure 2) showed significant difference $(\mathrm{P}<0.05)$ in the mean TG (mg/dl) of mild (M1- M2), moderate (M1-M2) and severe (M1- M2) anaemia groups were (173.28 $\pm 6.17-169.42 \pm 7.18),(167.02$ $\pm 14.82-163.95 \pm 14.06)$ and $(158.55 \pm 5.39-156.50 \pm 5.64)$ respectively. While contrast significant differences were observed for the other month among all anaemia groups. The levels of TG $(\mathrm{mg} / \mathrm{dl})$ before therapy (M1) and after therapy (M2-M7) showed significant difference $(\mathrm{P}<0.01)$ in mild anaemia $(173.28 \pm 6.17)$ and $(166.14 \pm 7.83 \mathrm{M} 3-$

\begin{tabular}{|c|c|c|c|}
\hline $\begin{array}{l}\text { Groups } \\
\text { Months }\end{array}$ & $\begin{array}{c}\text { Mild } \\
\text { (NO:36) } \\
\text { Mean } \pm \text { S.D. }\end{array}$ & $\begin{array}{c}\text { Moderate } \\
\text { (NO:62) } \\
\text { Mean } \pm \text { S.D. }\end{array}$ & $\begin{array}{c}\text { Severe } \\
\text { (NO:42) } \\
\text { Mean } \pm \text { S.D. }\end{array}$ \\
\hline M1 & $\begin{array}{c}173.28 \pm 6.17 \\
B C\end{array}$ & $\begin{array}{c}167.02 \pm 14.82 \\
\text { AC }\end{array}$ & $\begin{array}{c}158.55 \pm 5.39 \\
A B\end{array}$ \\
\hline M2 & $\begin{array}{c}169.42 \pm 7.18^{*} \\
\text { BC }\end{array}$ & $\begin{array}{c}163.95 \pm 14.06 \\
A C\end{array}$ & $\begin{array}{c}156.50 \pm 5.64 \\
A B\end{array}$ \\
\hline M3 & $166.14 \pm 7.83^{* *}$ & $\begin{array}{c}162.81 \pm 13.42 \\
C\end{array}$ & $\begin{array}{c}155.00 \pm 6.19 \text { * } \\
A B\end{array}$ \\
\hline M4 & ${ }_{\mathrm{C}}^{162.94 \pm 7.51}{ }^{* *}$ & $\begin{array}{c}161.61 \pm 13.26 \\
C\end{array}$ & $\begin{array}{c}152.07 \pm 6.08 \text { ** } \\
A B\end{array}$ \\
\hline M5 & ${ }_{\mathrm{C}}^{159.64 \pm 7.61}{ }^{* *}$ & $\begin{array}{c}159.60 \pm 13.58 \text { ** } \\
C\end{array}$ & $\begin{array}{c}149.55 \pm 6.48 \text { ** } \\
A B\end{array}$ \\
\hline M6 & $\frac{156.03 \pm 8.38 * *}{C}$ & $\begin{array}{c}157.40 \pm 13.42 \text { ** } \\
C\end{array}$ & $\begin{array}{c}146.79 \pm 6.00 \text { ** } \\
A B\end{array}$ \\
\hline M7 & $\begin{array}{c}152.64 \pm 8.57^{* *} \\
C\end{array}$ & $\begin{array}{c}154.03 \pm 13.31 \text { ** } \\
C\end{array}$ & $\begin{array}{c}143.81 \pm 6.15^{* *} \\
A B\end{array}$ \\
\hline
\end{tabular}

Table 4: Comparison of triglyceride between rhEPO responders patients.

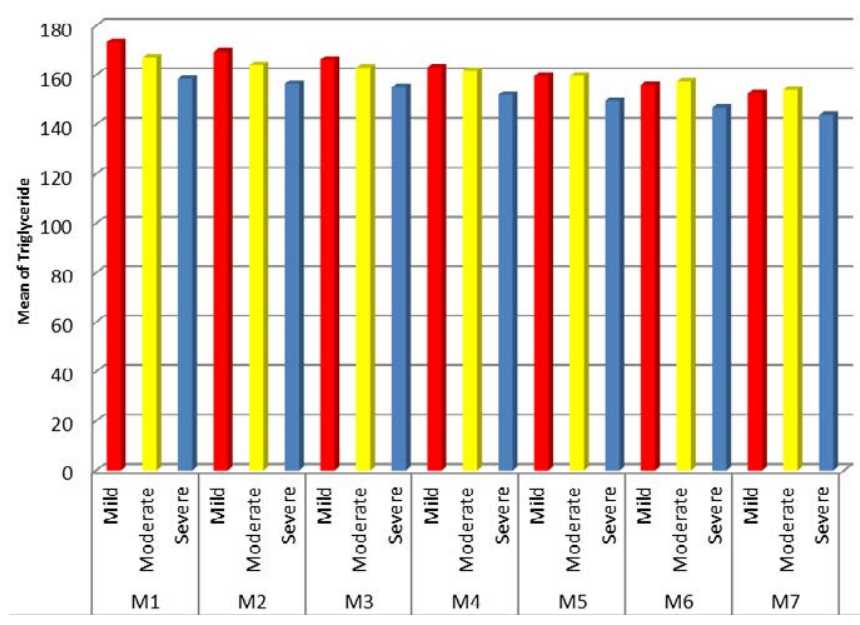

Figure 2: Comparison of triglyceride between rhEPO responders patients. 


\begin{tabular}{|c|c|c|c|}
\hline $\begin{array}{l}\text { Groups } \\
\text { Months }\end{array}$ & $\begin{array}{c}\text { Mild } \\
\text { (NO:36) } \\
\text { Mean } \pm \text { S.D. }\end{array}$ & $\begin{array}{c}\text { Moderate } \\
\text { (NO:62) } \\
\text { Mean } \pm \text { S.D. }\end{array}$ & $\begin{array}{c}\text { Severe } \\
\text { (NO:42) } \\
\text { Mean } \pm \text { S.D. }\end{array}$ \\
\hline M1 & $\begin{array}{c}36.89 \pm 1.91 \\
C\end{array}$ & $\begin{array}{c}35.21 \pm 2.24 \\
C\end{array}$ & $\begin{array}{c}32.50 \pm 8.57 \\
\mathrm{AB}\end{array}$ \\
\hline M2 & $\begin{array}{c}37.86 \pm 2.55 \\
C\end{array}$ & $\begin{array}{c}36.82 \pm 2.41^{* *} \\
C\end{array}$ & $\begin{array}{c}35.67 \pm 2.68 \text { * } \\
A B\end{array}$ \\
\hline M3 & $\begin{array}{c}39.19 \pm 3.18^{* *} \\
C\end{array}$ & $38.03 \pm 2.56$ ** & $\begin{array}{c}36.90 \pm 3.18^{* *} \\
\mathrm{~A}\end{array}$ \\
\hline M4 & $\begin{array}{c}41.25 \pm 3.40^{* *} \\
\text { BC }\end{array}$ & $\begin{array}{c}39.85 \pm 2.96 \text { ** } \\
\text { AC }\end{array}$ & $\begin{array}{c}37.57 \pm 2.89 \text { ** } \\
A B\end{array}$ \\
\hline M5 & $\begin{array}{c}44.22 \pm 4.22 \text { ** } \\
\text { BC }\end{array}$ & $\begin{array}{c}40.37 \pm 3.17^{* *} \\
\text { AC }\end{array}$ & $\begin{array}{c}37.76 \pm 3.27 \text { ** } \\
A B\end{array}$ \\
\hline M6 & $\begin{array}{c}48.00 \pm 4.96 \text { ** } \\
\text { BC }\end{array}$ & $\begin{array}{c}40.55 \pm 3.36 \text { ** } \\
\text { AC }\end{array}$ & $\begin{array}{c}38.24 \pm 3.26 \text { ** } \\
A B\end{array}$ \\
\hline M7 & $\begin{array}{c}51.28 \pm 5.17 \text { ** } \\
B C\end{array}$ & $\begin{array}{c}41.50 \pm 3.28 \text { ** } \\
\text { AC }\end{array}$ & $\begin{array}{c}38.79 \pm 2.95 \text { ** } \\
A B\end{array}$ \\
\hline
\end{tabular}

Table 5: Comparison of HDL between rhEPO responders patients.

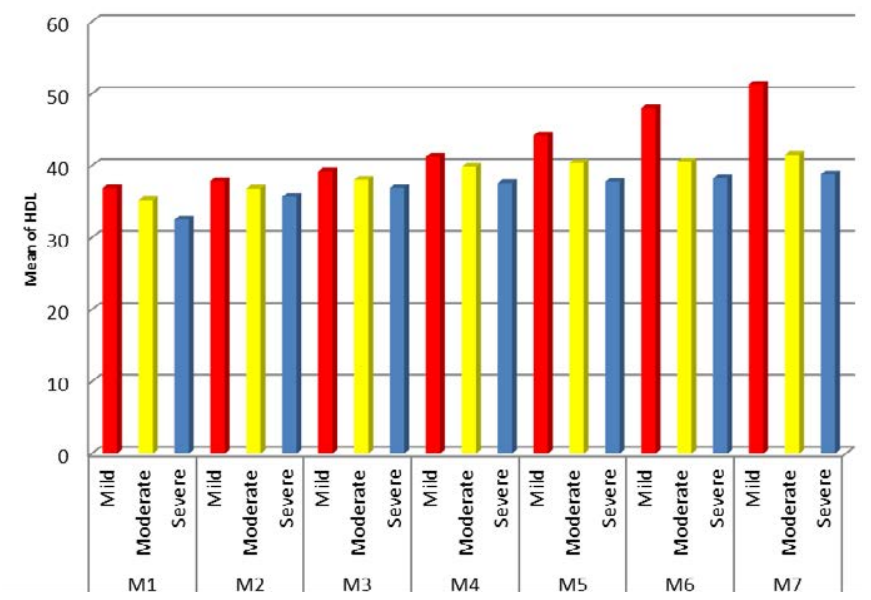

In the rhEPO non-responders results showed decrease in $\mathrm{Hb}(\mathrm{g} / \mathrm{dl})$ and Hct $(\%)$ levels .There was no change in TC (mg/dl), TG (mg/dl) and HDL-C $(\mathrm{mg} / \mathrm{dl})$ levels during the period of EPO therapy.

Figure 3: Comparison of HDL between rhEPO responders patients.

\begin{tabular}{|c|c|c|c|}
\hline Groups & $\begin{array}{c}\text { Mild } \\
\text { (NO:8) } \\
\text { Mean } \pm \text { S.D. }\end{array}$ & $\begin{array}{c}\text { Moderate } \\
\text { (NO:26) } \\
\text { Mean } \pm \text { S.D. }\end{array}$ & $\begin{array}{c}\text { Severe } \\
\text { (NO:26) } \\
\text { Mean } \pm \text { S.D. }\end{array}$ \\
\hline Months & $\begin{array}{c}10.89 \pm 0.87 \\
\text { BC }\end{array}$ & $\begin{array}{c}8.86 \pm 0.51 \\
\text { AC }\end{array}$ & $\begin{array}{c}6.46 \pm 0.56 \\
\text { AB }\end{array}$ \\
\hline M1 & $11.41 \pm 1.16$ & $9.39 \pm 0.66 * *$ & $7.96 \pm 0.62 * *$ \\
M2 & BC & AC & AB \\
\hline M3 & $9.90 \pm 1.07$ & $9.47 \pm 1.07 *$ & $8.36 \pm 1.41^{* *}$ \\
& C & C & AB \\
\hline M4 & $10.45 \pm 1.48$ & $9.66 \pm 1.43^{*}$ & $8.89 \pm 1.80 * *$ \\
& C & $8.64 \pm 1.48$ & $8.21 \pm 1.76 * *$ \\
\hline M5 & $9.95 \pm 1.17$ & $\mathrm{~A}$ & $\mathrm{~A}$ \\
\hline M6 & $9.08 \pm 1.36 * *$ & $8.05 \pm 1.10 * *$ & $7.36 \pm 1.53 * *$ \\
& $\mathrm{C}$ & & $\mathrm{A}$ \\
\hline M7 & $8.01 \pm 0.95 * *$ & $6.61 \pm 1.42 * *$ & $5.67 \pm 1.27^{* *}$ \\
& $\mathrm{C}$ & $\mathrm{C}$ & $\mathrm{AB}$ \\
\hline
\end{tabular}

Table 6: Comparison of $\mathrm{Hb}$ between rhEPO non-responder patients.

$152.64 \pm 8.57 \mathrm{M} 7)$, moderate anaemia $(167.02 \pm 14.82)$ and $(159.60$ $\pm 13.58 \mathrm{M} 5-154.03 \pm 13.31 \mathrm{M} 7)$ and severe anaemia groups $(158.55 \pm$ $5.39)$ and $(155.00 \pm 6.19 \mathrm{M} 3-143.81 \pm 6.15 \mathrm{M} 7)$ respectively.

Table 5 and Figure 3 showed significant difference $(\mathrm{P}<0.05)$ in the mean HDL (mg/dl) of mild (M4- M7), moderate (M4-M7) and severe
(M4- M7) anaemia groups were $(41.25 \pm 3.40-51.28 \pm 5.17),(39.85 \pm$ $2.96-41.50 \pm 3.28)$ and $(37.57 \pm 2.89-38.79 \pm 2.95)$ respectively. While significant differences were observed for the other months among all anaemia groups. The levels of HDL (mg/dl) before therapy (M1) and after therapy $(\mathrm{M} 2-\mathrm{M} 7)$ showed significant difference $(\mathrm{P}<0.01)$ in patients with mild anaemia $(36.89 \pm 1.91)$ and $(39.19 \pm 3.18 \mathrm{M} 3-51.28$ $\pm 5.17 \mathrm{M} 7)$, moderate anaemia $(35.21 \pm 2.24)$ and $(36.82 \pm 2.41 \mathrm{M} 2-$ $41.50 \pm 3.28 \mathrm{M} 7)$ and severe anaemia groups $(32.50 \pm 8.57)$ and $(36.90$ $\pm 3.18 \mathrm{M} 3-38.79 \pm 2.95$ ) M7 respectively (Tables 6-10).

\begin{tabular}{|c|c|c|c|}
\hline $\begin{array}{l}\text { Groups } \\
\text { Months }\end{array}$ & $\begin{array}{c}\text { Mild } \\
\text { (NO:8) } \\
\text { Mean } \pm \text { S.D. }\end{array}$ & $\begin{array}{c}\text { Moderate } \\
\text { (NO:26) } \\
\text { Mean } \pm \text { S.D. }\end{array}$ & $\begin{array}{c}\text { Severe } \\
\text { (NO:26) } \\
\text { Mean } \pm \text { S.D. }\end{array}$ \\
\hline M1 & $\begin{array}{c}32.13 \pm 5.19 \\
B C\end{array}$ & $\begin{array}{c}28.31 \pm 1.38 \\
A C\end{array}$ & $\begin{array}{c}21.96 \pm 0.99 \\
A B\end{array}$ \\
\hline M2 & $\begin{array}{c}34.75 \pm 3.41 \\
B C\end{array}$ & $\begin{array}{c}30.04 \pm 2.03 \text { ** } \\
A C\end{array}$ & $\begin{array}{c}25.46 \pm 2.04 \text { ** } \\
A B\end{array}$ \\
\hline M3 & $\begin{array}{c}31.75 \pm 3.88 \\
C\end{array}$ & $\begin{array}{c}30.31 \pm 2.98^{* *} \\
C\end{array}$ & $\begin{array}{c}29.25 \pm 3.86 \text { ** } \\
A B\end{array}$ \\
\hline M4 & $\begin{array}{c}33.00 \pm 4.44 \\
C\end{array}$ & $30.92 \pm 4.39$ ** & $\begin{array}{c}29.04 \pm 4.94 \text { ** } \\
\text { * }\end{array}$ \\
\hline M5 & $\begin{array}{c}31.50 \pm 4.04 \\
C\end{array}$ & $28.00 \pm 4.55$ & $\begin{array}{c}26.81 \pm 5.28 \text { ** } \\
A\end{array}$ \\
\hline M6 & $\begin{array}{c}28.63 \pm 3.70 \\
C\end{array}$ & $25.96 \pm 3.12$ * & $\frac{24.12 \pm 4.60}{\mathrm{~A}}$ * \\
\hline M7 & $\begin{array}{c}25.38 \pm 2.62 * * \\
C\end{array}$ & $\begin{array}{c}23.46 \pm 2.76 \text { ** } \\
C\end{array}$ & $\begin{array}{c}19.00 \pm 4.55^{\star *} \\
A B\end{array}$ \\
\hline
\end{tabular}

Table 7: Comparison of Hct between rhEPO non-responder patients.

\begin{tabular}{|c|c|c|c|}
\hline $\begin{array}{l}\text { Groups } \\
\text { Months }\end{array}$ & $\begin{array}{c}\text { Mild } \\
(\text { NO:8) } \\
\text { Mean } \pm \text { S.D. }\end{array}$ & $\begin{array}{c}\text { Moderate } \\
\text { (NO:26) } \\
\text { Mean } \pm \text { S.D. }\end{array}$ & $\begin{array}{c}\text { Severe } \\
\text { (NO:26) } \\
\text { Mean } \pm \text { S.D. }\end{array}$ \\
\hline M1 & $\begin{array}{c}252.13 \pm 22.34 \\
B C\end{array}$ & $\begin{array}{c}231.65 \pm 10.27 \\
A C\end{array}$ & $\begin{array}{c}173.77 \pm 26.88 \\
A B\end{array}$ \\
\hline M2 & $\begin{array}{c}249.00 \pm 20.06 \\
C\end{array}$ & $\begin{array}{c}231.04 \pm 9.67 \\
C\end{array}$ & $\begin{array}{c}174.42 \pm 28.56 \\
A B\end{array}$ \\
\hline M3 & $\begin{array}{c}251.38 \pm 19.72 \\
B C\end{array}$ & $\begin{array}{c}230.46 \pm 11.69 \\
A C\end{array}$ & $\begin{array}{c}174.50 \pm 28.81 \\
A B\end{array}$ \\
\hline M4 & $\begin{array}{c}249.51 \pm 18.44 \\
B C\end{array}$ & $\begin{array}{c}230.46 \pm 12.43 \\
A C\end{array}$ & $\begin{array}{c}172.58 \pm 30.17 \\
A B\end{array}$ \\
\hline M5 & $\begin{array}{c}248.38 \pm 20.43 \\
B C\end{array}$ & $\begin{array}{c}229.96 \pm 13.83 \\
A C\end{array}$ & $\begin{array}{c}170.81 \pm 31.99 \\
A B\end{array}$ \\
\hline M6 & $\begin{array}{c}245.91 \pm 26.89 \\
B C\end{array}$ & $\begin{array}{c}227.01 \pm 13.74 \\
A C\end{array}$ & $\begin{array}{c}168.04 \pm 34.51 \\
A B\end{array}$ \\
\hline M7 & $\begin{array}{c}243.1 \pm 31.21 \\
B C\end{array}$ & $\begin{array}{c}223.34 \pm 12.76 \\
A C\end{array}$ & $\begin{array}{c}165.08 \pm 37.56 \\
A B\end{array}$ \\
\hline
\end{tabular}

Table 8: Comparison of cholesterol between rhEPO non-responders patients.

\begin{tabular}{|c|c|c|c|}
\hline Groups & $\begin{array}{c}\text { Mild } \\
\text { (NO:8) } \\
\text { Mean } \pm \text { S.D. }\end{array}$ & $\begin{array}{c}\text { Moderate } \\
\text { (NO:26) } \\
\text { Mean } \pm \text { S.D. }\end{array}$ & $\begin{array}{c}\text { Severe } \\
\text { (NO:26) } \\
\text { Mean } \pm \text { S.D. }\end{array}$ \\
\hline Months & $167.50 \pm 6.82$ & $165.92 \pm 7.59$ & $145.73 \pm 7.88$ \\
M1 & C & C & AB \\
\hline M2 & $167.58 \pm 5.63$ & $165.54 \pm 8.06$ & $144.00 \pm 7.40$ \\
& C & C & AB \\
\hline M3 & $167 \pm 4.58$ & $165.73 \pm 7.34$ & $144.38 \pm 7.83$ \\
& C & C & AB \\
\hline M4 & $167 \pm 10.03$ & $165.88 \pm 8.94$ & $143.54 \pm 8.44$ \\
& C & C & AB \\
\hline M5 & $166.88 \pm 10.83$ & $164.00 \pm 9.35$ & $143.34 \pm 8.63$ \\
& BC & AC & AB \\
\hline M6 & $166.53 \pm 12.46$ & $164.58 \pm 9.67$ & $143.38 \pm 7.82$ \\
& BC & AC & AB \\
\hline M7 & $166 \pm 14.99$ & $163.42 \pm 9.74$ & $142.52 \pm 8.15$ \\
& $\mathrm{BC}$ & AC & AB \\
\hline
\end{tabular}

Table 9: Comparison of triglyceride between rhEPO non-responders patients 


\begin{tabular}{|c|c|c|c|}
\hline Groups & $\begin{array}{c}\text { Mild } \\
\text { (NO:8) } \\
\text { Mean } \pm \text { S.D. }\end{array}$ & $\begin{array}{c}\text { Moderate } \\
\text { (NO:26) } \\
\text { Mean } \pm \text { S.D. }\end{array}$ & $\begin{array}{c}\text { Severe } \\
\text { (NO:26) } \\
\text { Mean } \pm \text { S.D. }\end{array}$ \\
\hline M1 & $36.75 \pm 1.04$ & $36.00 \pm 0.94$ & $35.12 \pm 0.99$ \\
& $\mathrm{C}$ & $\mathrm{C}$ & $\mathrm{AB}$ \\
\hline M2 & $36.88 \pm 0.83$ & $36.01 \pm 1.13$ & $35.15 \pm 0.78$ \\
& $\mathrm{C}$ & $\mathrm{C}$ & $\mathrm{AB}$ \\
\hline M3 & $37.00 \pm 1.85$ & $36.25 \pm 1.17$ & $35.21 \pm 1.34$ \\
& $\mathrm{C}$ & $\mathrm{C}$ & $\mathrm{AB}$ \\
\hline M4 & $37.21 \pm 3.49$ & $36.41 \pm 1.59$ & $35.34 \pm 1.80$ \\
& $\mathrm{C}$ & $\mathrm{C}$ & $\mathrm{AB}$ \\
\hline M5 & $37.35 \pm 2.71$ & $36.63 \pm 1.81$ & $35.5 \pm 1.68$ \\
& $\mathrm{BC}$ & $\mathrm{AC}$ & $\mathrm{AB}$ \\
\hline M6 & $37.35 \pm 1.31$ & $36.30 \pm 1.79$ & $34.95 \pm 1.99$ \\
& $\mathrm{BC}$ & $\mathrm{AC}$ & $\mathrm{AB}$ \\
\hline M7 & $37.10 \pm 1.41$ & $35.69 \pm 2.04$ & $34.65 \pm 3.54$ \\
& $\mathrm{BC}$ & $\mathrm{AC}$ & $\mathrm{AB}$ \\
\hline
\end{tabular}

Table 10: Comparison of HDL between rhEPO non-responders patients.

\section{Discussion}

In our study, cholesterol was lower in rhEPO responders than rhEPO non - responders patients, also cholesterol level is positively associated with improvement of anemia in CKD patients. This is similar with Tanaka et al., Manitius et al., Mak et al. and Ponnudhali and Nagarajan [14-18] showed that EPO therapy is associated with an improvement in the blood lipid profile especially cholesterol and LDL $\mathrm{C}$ in CKD patients and in complement with Allegra et al. and Nissenson and Fine $[19,20]$ suggested that long - term treatment with EPO altered total serum cholesterol and LDL - C in patients. However, this effect is influenced significantly by food intake.

Triglyceride was lower in rhEPO responders than rhEPO non responders patients, also TG level is positively associated with EPO therapy in CKD patients. This is similar with Pollock et al., Jamil and Naqvi, Bugeja and Chan and Naini et al. [21-24] showed that long term rhEPO treatment is associated with an improvement in the TG levels.

High density lipoprotein was higher in rhEPO responders than rhEPO non - responders patients, also HDL level is positively associated with EPO therapy in CKD. This is compatible with other studies Papavasiliou et al, Siamopoulos et al. and Tselepis and Siamopoulos [25-27] suggested that, EPO treatment of predialysis patients with CKD significantly increases serum HDL - C levels and HDL - C / LDL - C, which may represent an important anti atherogenic effect of this hormone. In CKD, epoetin alfa improves quality of life, physical activity and increased tissue oxygenation that lead to an increase in activity of several enzymes and transferring proteins involved in HDL-C biogenesis as well as in HDL maturation and lead to the increase in HDL-C levels [27,28].

\section{References}

1. Sarnak MJ, Levey AS (2000) Cardiovascular disease and chronic renal disease: a new paradigm. Am J Kidney Dis 35: S117-131.

2. Weiner DE, Tighiouart H, Amin MG, Stark PC, MacLeod B, et al. (2004) Chronic kidney disease as a risk factor for cardiovascular disease and all-cause mortality: a pooled analysis of community-based studies. J Am Soc Nephrol 15: 1307-1315.

3. Warady B, Schaefer J, Alexander S (2012) Pediatric Dialysis (2ndedn) Springer, USA, 506.

4. Kasiske BL (1998) Hyperlipidemia in patients with chronic renal disease. Am J Kidney Dis 32: S142-156.

5. Weiner DE, Sarnak MJ (2004) Managing dyslipidemia in chronic kidney disease. J Gen Intern Med 19: 1045-1052.
6. Mancini M, Ordovas J, Riccardi G, Rubba P, Strazzullo P (2011) Nutritional and Metabolic Bases of Cardiovascular Disease (1stedn) Wiley-Blackwell 488

7. Miller M, Stone NJ, Ballantyne C, Bittner V, Criqui MH, et al. (2011) Triglycerides and cardiovascular disease: a scientific statement from the American Heart Association. Circulation 123: 2292-2333.

8. Lerma E and Nissenson A (2011) Nephrology Secrets (3rdedn) Elsevier 3-4.

9. Henrich W (2012) Principles and Practice of dialysis (4thedn) Lippincott Williams \& Wilkins P.375

10. Castelli WP, Garrison RJ, Wilson PW, Abbott RD, Kalousdian S, et al. (1986) Incidence of coronary heart disease and lipoprotein cholesterol levels. The Framingham Study. JAMA 256: 2835-2838.

11. Foody J (2007) Preventive Cardiology: Insights into the prevention and treatment of Cardiovascular disease (2nd edn) Springer USA, 82.

12. Toth PP (2005) High-density lipoprotein as a therapeutic target: clinica evidence and treatment strategies. Am J Cardiol 96: 50K-58K.

13. Assmann G, Gotto AM Jr (2004) HDL cholesterol and protective factors in atherosclerosis. Circulation 109: III8-14.

14. Tanaka H, Kan E, Takegaki Y, Inariba H, Yoshimoto M, et al. (1993) Multicenter study with recombinant human erythropoietin. Artif Organs 17: 213-218.

15. Manitius J, SzoÅ,kiewicz M, MyÅ>liwska J, Zorena K, Myśliwski A, et al. (1995) Influence of 'nonhematological' doses of erythropoietin on lipid-carbohydrate metabolism and life quality in hemodialysis patients. Nephron 69: 363-364

16. Mak RH (1996) Effect of recombinant human erythropoietin on insulin, amino acid, and lipid metabolism in uremia. J Pediatr 129: 97-104

17. Kes P, Bobić I, Reiner Z, Ratković-Gusić I (2002) [Effect of erythropoietin therapy on serum lipoprotein levels in patients on hemodialysis]. Lijec Vjesn 124: $146-150$.

18. Ponnudhali D, Nagarajan P (2011) Lipoprotein (a) and dyslipidemia in predialysis chronic kidney disease patients and in patients on maintenance hemodialysis. International Journal of Basic Medical Science 2: 131-137.

19. Allegra V, Martimbianco L, Vasile A (1997) Lipid and apolipoprotein patterns during erythropoietin therapy: roles of erythropoietin, route of administration, and diet. Nephrol Dial Transplant 12: 924-932.

20. Nissenson A, Fine R (2008) Handbook of Dialysis Therapy (4thedn) Elsevier 1632.

21. Pollock CA, Wyndham R, Collett PV, Elder G, Field MJ, et al. (1994) Effects of erythropoietin therapy on the lipid profile in end-stage renal failure. Kidney Int 45: 897-902

22. Jamil B, Naqvi S (1996) Effect of erythropoietin on lipid profile in haemodialysis patients. Nephrology 2: 437-438.

23. Bugeja AL, Chan CT (2004) Improvement in lipid profile by nocturnal hemodialysis in patients with end-stage renal disease. ASAIO J 50: 328-331.

24. Emami Naini A, Moradi M, Mortazavi M, Amini Harandi A, Hadizadeh M, et al (2012) Effects of Oral L-Carnitine Supplementation on Lipid Profile, Anemia and Quality of Life in Chronic Renal Disease Patients under Hemodialysis: A Randomized, Double-Blinded, Placebo-Controlled Trial. Journal of Nutrition and Metabolism 1-6.

25. Papavasiliou EC, Gouva C, Siamopoulos KC, Tselepis AD (2006) PAFacetylhydrolase activity in plasma of patients with chronic kidney disease. Effect of long-term therapy with erythropoietin. Nephrol Dial Transplant 21: 1270-1277.

26. Siamopoulos KC, Gouva C, Katopodis KP, Tzallas C, Nikolopoulos P, et al. (2006) Long-term treatment with EPO increases serum levels of high-density lipoprotein in patients with CKD. Am J Kidney Dis 48: 242-249.

27. Tselepis A, Siamopoulos K (2007) Effect of Long-term Epoetin Treatment on Serum Levels of High-density Lipoprotein (HDL) and HDL-associated Enzymes in Patients with Chronic Kidney Disease. European Nephrology 2: 34-36.

28. National Cholesterol Education Program (NCEP) Expert Panel on Detection Evaluation, and Treatment of High Blood Cholesterol in Adults (Adult Treatment Panel III) (2002) Third Report of the National Cholesterol Education Program (NCEP) Expert Panel on Detection, Evaluation, and Treatment of High Blood Cholesterol in Adults (Adult Treatment Panel III) final report. Circulation 106 3143-3421. 